\title{
A Coarse-to-Fine Registration Method Based on Geometric Constraints and Parallel Architecture
}

\author{
Zetao Jiang1, Chuan Guo ${ }^{2 *}$, Yang Yang ${ }^{2}$ \\ 1 Guilin University of Electronic Technology, Guilin, China. Nanchang Hangkong University, Nanchang, China \\ 2 Nanchang Hangkong University, Nanchang, China \\ * Corresponding author. Tel.: (+86)15070998803; email: nchuguochuan@126.com. \\ Manuscript submitted July 30, 2014; accepted April10, 2015. \\ doi: 10.17706/jsw.10.5.551-565
}

\begin{abstract}
Aiming at solving lengthy time-consuming registration and mismatch problems caused by images which have feature simliarity of local region in the traditional SIFT (Scale Invariant Feature Transform) registration method, a fine registration method based on geometric constraints and parallel architecture is put forward. First, in order to provide geometric constraints for block and fine registration, we use SIFT algorithm parallelly extract feature points to calculate initial transform matrix. Then, segment the overlapping region of original image pairs into several blocks. Finally, to achieve fine registration, blockwise SIFT matching and eliminate the mismatch through geometric constraints are implemented on parallel architecture. During the fine registration, we use geometric constraints and affine invariance of Mahalanobis distance to screen feature points and eliminate duplicate matches and mismatches. The experimental results show that this method has eliminated the mismatch generated by same local features. Moreover, registration accuracy and speed have been improved, the proposed approach has practical value.
\end{abstract}

Key words: Image registration, geometric constraints, affine invariant, block strategy, parallel.

\section{Introduction}

Image registration and fusion splicing technology are the hot issues of the image processing, machine vision and computer graphics research. Registration have a wide range of applications in image fusion, video monitoring, automatic target recognition, biomedical analysis, field of virtual reality, military, and remote sensing technology etc. Image matching is putting several images of the same scene with overlap sequence into a high resolution image with big size and wide angle of view. Image registration [1]-[3] is the key technology in image mosaic, and it affects the precision and speed of image stitching. Recently the most widely used registration method is based on feature points matching [4]-[9]. This is a method that extracts the feature points in the images through implementing feature detection from paired image firstly, and then completes image registration after generating feature descriptors.

C. Harris and M. J. Stephens [10] proposed the famous Harris operator. Harris corner is invariant to noise, rotation and illumination, but it is sensitive to scale changes. So it was not used in the multi-scale image registration. Lowe [11] proposed a landmark local feature based on the theory of scale space, i.e., the famous SIFT (Scale Invariant Feature Transform) operator. SIFT is invariant to scale, rotation, transformation, and illumination changes of image. But the dimensions of the SIFT feature descriptor is very high, and it is not applicable to real-time demand higher occasion. After that, SURF [12], [13], SSIFT [14] and PCA-SIFT [15], [16] algorithms are effective accelerating for the SIFT. Moreover these algorithms 
improve the efficiency of the registration, but their accuracies are worse than that of SIFT algorithm. Accordingly we choose SIFT points to get initial matching. The experimental results show that this method produces some mismatch problems which resulted by local region similar or SIFT vector similar although the SIFT feature point accuracy is high, as shown as the red box marked part in Fig. 1. Ji Hua [17] who proposed a combination of global information SIFT feature matching algorithm, solved the mismatch problem caused by local region similar to a certain extent. However, this method increases the amount of calculation and it is sensitive to scale changes. Bastanlar [18] proposed an improved SIFT algorithm to solve the large scale difference problem of image registration and improved registration accuracy. However, the method does not solve the above problem of mismatch. Goncalves [19] proposed a image registration method based on the combination of image segmentation and SIFT to eliminate the mismatch, however, this method cannot be applied to the occasion which has higher demand for time response. Qiang Shi [20] proposed a novel method of image registration to overcome similar patterns and brightness changes. But this is difficult for registering distorted images and the efficiency of this method is not high. Kai Zhang [21] proposed a highly robust matching algorithm, which is based on the feature point descriptor calculated by the TAR (triangle-area representation) of the K nearest neighbors (KNN-TAR). The proposed method can remove the outliers from the initial matching result even when the outliers are of high proportion. But the calculation of the value of KNN-TAR is complex, the algorithm cannot be applied to the occasion which has higher demand for time response.

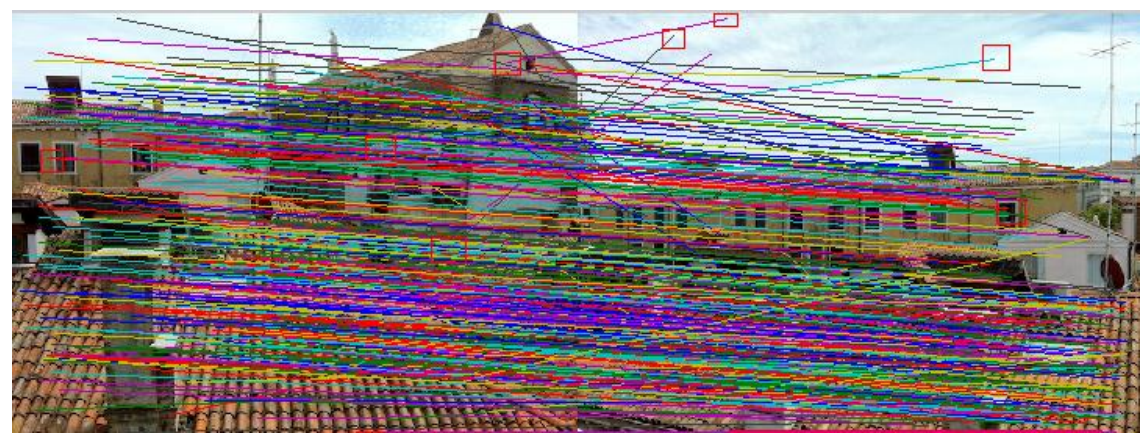

Fig. 1. Mismatch generated by same local features.

This paper has proposed an efficient coarse-to-fine registration method on the basis of geometric constraints and parallel architecture to solve two problems: Mismatch problems caused by the feature simliarity among local region of images, and the time-consuming registration. First of all, in order to provide geometric constraints for block and fine registration, we use SIFT algorithm parallelly extract feature points to calculate initial transform matrix. Secondly, segment the overlapping region of original image pairs into several blocks. And then, to achieve fine registration, blockwise SIFT matching and eliminate the mismatch through geometric constraints are implemented on parallel architecture. During the fine registration, we use geometric constraints and affine invariance of Mahalanobis distance to screen feature points further and eliminate duplicate matches and mismatches to improve the matching accuracy. Next, outlier removal [22] is done after finishing fine registration. Finally, we got the image mosaic result through transformation estimation and image resampling [23].

\section{Multi-Scale Feature Detection and Initial Matching}

\subsection{IFT Point Detection}

The SIFT algorithm first constructs Gaussian pyramid, the scale space $L(x, y, \delta)$ of image $I(x, y)$ will be constructed by convolution of image $I(x, y)$ and Gaussian kernel function. That is, $\delta$ is the scale factor of 
Gaussian function, in order to control the level of image's smoothness, we can change the size of the $\delta$. We intend to construct $O$ octaves, $S$ levels images and appoint scale value $\delta$ for each image defined as below:

$$
\begin{gathered}
L(x, y, \delta)=G(x, y, \delta) \times I(x, y), \\
G(x, y, \delta)=\frac{1}{2 \pi \delta^{2}} e^{-\left(x^{2}+y^{2}\right) / 2 \delta^{2}}, \\
\delta(o, s)=2^{o-1} \times k^{s} \times \delta_{0},
\end{gathered}
$$

where $\delta_{0}$ means initial scale value, $o$ and $s$ represent $o t h$, sth image is proportional constant of the scale factor.

$$
k=2^{1 / S} \text {, }
$$

Lowe[11] experimentally found detecting extreme points on the Difference of Gaussian scale-space can efficiently extract stable feature point candidates. Then, Gaussian $D o G$ images will be obtained by $D o G$ operator:

$$
D(x, y, \delta)=[G(x, y, k \delta)-G(x, y, \delta)] \times I(x, y) .
$$

The process of SIFT algorithm: firstly, construct Gaussian pyramid ( $O$ octaves, $S$ levels images). And then construct Difference of Gaussian space, feature points are made up of 3-D local extreme points of Difference of Gaussian images. Finally, after detecting extreme points we can't regard them as SIFT points until the heavy edge corresponding is eliminated through the Hessian matrix.

\subsection{SIFT Feature Descriptor}

Feature descriptor is used to describe the gradient statistics of the feature point neighborhood, it is unique. SIFT descriptor can be obtained by gradient histogram of $16 \times 16$ around the feature point. As show in Fig. 2, First in order to ensure the scale invariance of feature descriptor, we compute the feature descriptor on Gaussian blur image $L(x, y, \delta)$ that scale is equal to feature point. In order to guarantee the rotation invariance of feature descriptor, we compute the main orientation of feature point. And then we divide $16 \times 16$ into $4 \times 4$ small regions with the size $4 \times 4$. Next, we make a statistics about 8 gradient histogram of every $4 \times 4$ region and obtain the descriptor vector whose dimension is $4 \times 4 \times 8=128$. In order to eliminate the influence of illumination differences, we normalize the vector. So far, the descriptor describes the change of gradient orientation around the feature point. Therefore it is robust to illumination, scale and rotation. Due to the elimination of edge corresponding, it is also robust to noise.
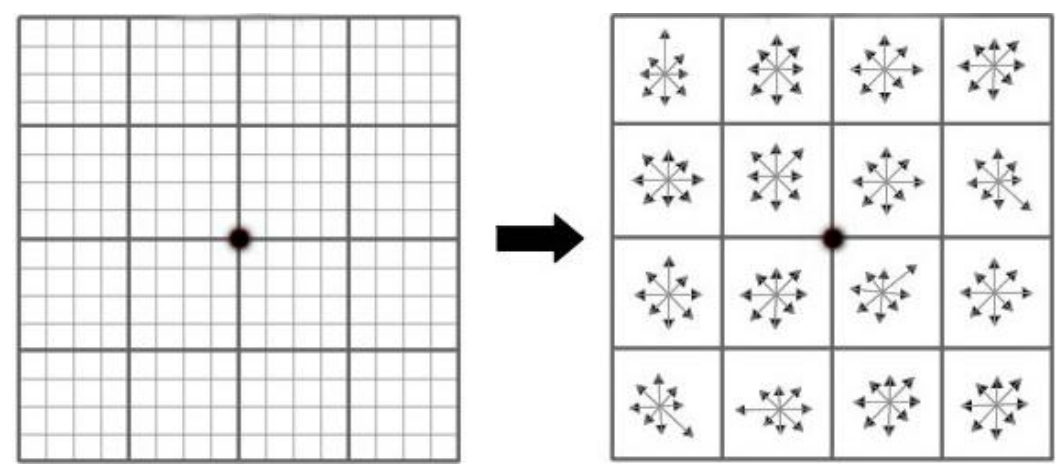

Fig. 2. The $16 \times 16$ window that computes feature point descriptor. 


\subsection{Initial Matching of Feature Points}

After the detection and description of features, we utilize Euclidean distance and BBF(Best Bin First) to get initial matching pairs. Let $P_{a}$ and $P_{b}$ be SIFT point set to be matched, SIFT point set $P_{a}$ is derived from the image $I_{L}$ and SIFT point set $P_{b}$ is derived from the image $I_{R}$. We get initial matching by following steps:

1) Segment feature point set $P_{b}$ and construct complete K-D tree.

2) For one point $a_{i}(i=1,2 \ldots n)\left(a_{i} \in P_{a}\right)$ in $P_{a}$, we find two feature points (Nearest Neighbor/Next nearest neighbor) ${ }^{b_{j}}$ and $b_{k}(j, k=1,2 \ldots m ; j \neq k)\left(b_{j} \in P_{b}, b_{k} \in P_{b}\right)$ in K-D tree, and then we get the two distance Dis $_{\text {nearest }}$ and $D i s_{\text {nextneares }}$.

3) Compute the ratio:

$$
\text { ratio }=\frac{D_{\text {nearest }}}{D_{\text {nextnearest }}},
$$

if ratio $<T_{\text {ratio }}$ (the recommended value is set between 0.4 and 0.65 , in this paper, $T_{\text {ratio }}=0.5$ ), $a_{i}$ and $b_{j}$ are matched, add the pair of match points $\left[a_{i}, b_{j}\right]$ to the corresponding matching point set $Z$, go to step 4. Otherwise, Skip to step2 after $i+1$, compute the matching point of next feature point $a_{i+1}\left(a_{i+1} \in P_{a}\right)$.

4)Output the set of feature matching until all points in $P_{a}$ are computed. Otherwise, Skip to step2 after $i+1$.

\section{Proposed Approach}

\subsection{Regional Division and Geometric Constraints}

The purpose of coarse matching is to calculate the initial matching transformation matrix $T$, and it can provide geometric constraints for regional division and fine registration. This step does not need too many feature points. In order to improve the efficiency of computation, we downsample the source images before doing the coarse registration. So as to prevent the excessive lose of image details, the downsampling rate should not be too large. For example, for a $4000 \times 4000$ image pair, $r=4$ is a good choice. In this paper, $r=2$. The downsampling rate is defined as follow:

$$
\begin{gathered}
r=\min \left(2^{n}, 8\right), \\
n=\left\lfloor\log _{2}\left(M_{s} / M_{d}\right)\right\rfloor, \\
M_{s}=\min \left(W_{s}, H_{s}\right), \\
M_{d}=\min \left(W_{d}, H_{d}\right) .
\end{gathered}
$$

where the $W_{s}$ and the $H_{s}$ are the width and height of the source image, the $W_{d}$ and the $H_{d}$ are the width and height of the image after downsampling.

According to the method of section II, we can implement coarse registration and calculate the initial transformation matrix $T$. With the help of the basis transformation matrix and geometric constraint relation of matching points, we can determine the overlapping area in the source image. Then divide the overlapping area into blocks. At last, fine registration is implemented in the regional blocks parallelly. As shown in Fig. 3 (the left image is a reference image, the right image is the one to be registered), determine the overlapping region in the reference image firstly: with the help of the initial transformation matrix $\mathrm{T}$ obtained at the coarse registration step, we can map the top left corner and the bottom left corner points ( $c$ and $d$ ) of the right image to the left image, i.e. 


$$
\begin{aligned}
c^{\prime} & =T(c), \\
d^{\prime} & =T(d) .
\end{aligned}
$$

By this way, we obtain the overlapping region $\left(R_{c^{\prime} d^{\prime} b a}\right)$ in reference image. In the same way, we map the top right corner and the bottom right corner points $(a$ and $b$ ) of the left image to the right image through the inverse transformation of T, i.e.,

$$
\begin{aligned}
& a^{\prime}=(\operatorname{inverse}(T))(a), \\
& b^{\prime}=(\operatorname{inverse}(T))(b),
\end{aligned}
$$

And we acquire the overlapping region $\left(R_{c d b^{\prime} a^{\prime}}\right)$ in source image. Here, these points $\left(c^{\prime}, d^{\prime}, a^{\prime}, b^{\prime}\right)$ might not on the edge of image, in order to facilitate subsequent processing, we adjust them to the edge coordinates.

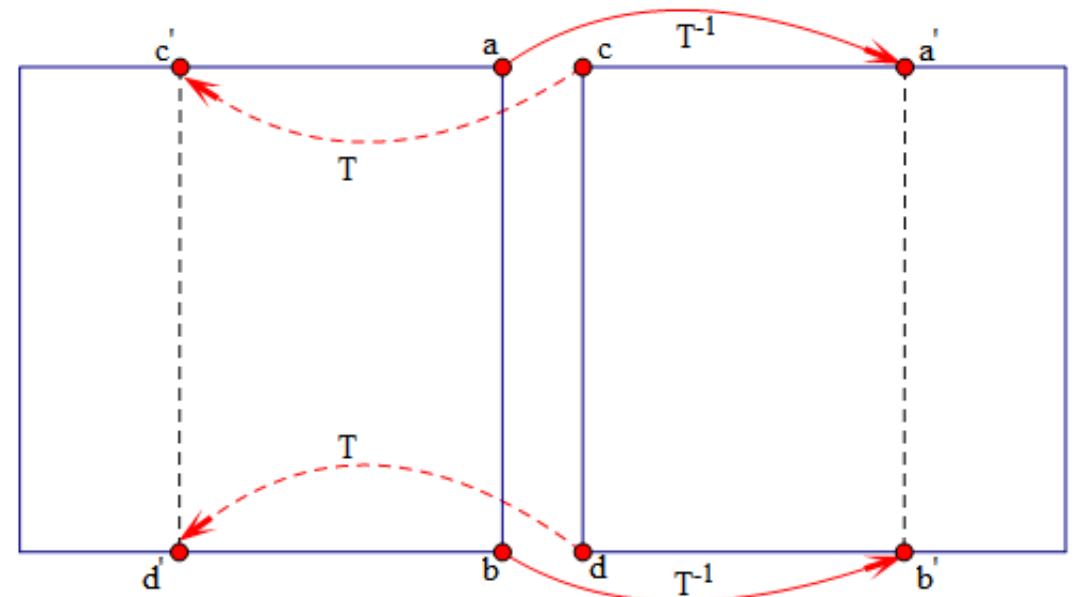

Fig. 3. Determine overlapping regions of the reference image and the image to be registered.

After determining the overlapping area, we segment the region according to the size of overlapping area: 1) If the overlapping area is more than $80 \%$ of the original image, region division of $3 \times 3$ template is implemented on the original image pairs. 2) If the overlapping area is more than $40 \%$ and less than $80 \%$ of the original image, region division of $3 \times 2$ template is implemented on the overlapping area of original image pairs. 3) If the overlapping area is less than $40 \%$ of the original image, region division of $3 \times 1$ template is implemented on the overlapping area of original image pairs. This approach has two advantages: on the one hand, if registration image pairs has obvious non-overlapping region, this method not only saves memory space but also reduces the unnecessary calculation thereby improves the efficiency. On the other hand, with the help of the constraint of block and the geometric constraint of the initial transformation matrix, the mismatch generated by same local features is eliminated and the matching accuracy is improved. As shown in Fig. 5, assume that descriptors of these feature points $\left(p_{1}, p_{2}, p_{3}, p_{4}\right)$ are similar. For $p_{1}, p_{2}, p_{4}$ these three feature points, due to the influence of noise and repetitive structure(same local features), the Euclidean distance between feature point $p_{1}$ and $p_{4}$ may be less than (or equal to) the Euclidean distance between $p_{1}$ and $p_{2}$. Hence, traditional SIFT registration method causes a mismatch $\left(p_{1}, p_{4}\right)$ in this occasion. The corresponding matching point of the feature point $p_{l}$ in the $R_{l}$ region may only appear in the corresponding region $R_{2}$, so proposed method no longer searches matching point in the region of $R_{3}$ through the constraint of region block, and the mismatch $\left(p_{1}, p_{4}\right)$ is removed 
effectively. For $p_{1}, p_{2}, p_{3}$ these three feature points, owing to the influence of repetitive structure(same local features) and noise, the Euclidean distance between $p_{1}$ and $p_{3}$ may be less than (or equal to) the Euclidean distance between $p_{1}$ and $p_{2}$. Therefore, traditional SIFT registration method causes a mismatch $\left(p_{1}, p_{3}\right)$ in this case. With the help of the geometric constraint which is provided by the initial transformation matrix, the mismatch is eliminated by following steps. Firstly, we map the $p_{3}$ to the reference image through the initial transformation matrix $T$. Then, the distance between them is calculated, i.e.,

$$
d=\operatorname{dis}\left(T\left(p_{3}\right), p_{1}\right) .
$$

Finally, if $d \geq \tau$, The mismatch $\left(p_{1}, p_{3}\right)$ is removed successfully. Where $\tau$ is a threshold, the value of $\tau$ affects the matching rate. We use Fig. 7(b) as an experiment images to test the influence of different threshold for matching rate, results (initial matches and matching rate) corresponding to different values of $\tau$ are shown in Fig. 4. The number of initial matches increases with the increase of $\tau$, whereas matching rate decreases with the increase of $\tau$. This is because in the process of threshold increases, we mistook the incorrect matches for match points. Therefore, the threshold cannot choose too high. On the other hand, the threshold value cannot be too low, and if low, the number of initial matches will reduced because the correct matching is filtered out. We see from the result of the experiment, when the threshold is between 60 to 120 , the change of the matching rate is relatively smooth. In order to obtain more initial matches, in this paper, $\tau=100$.

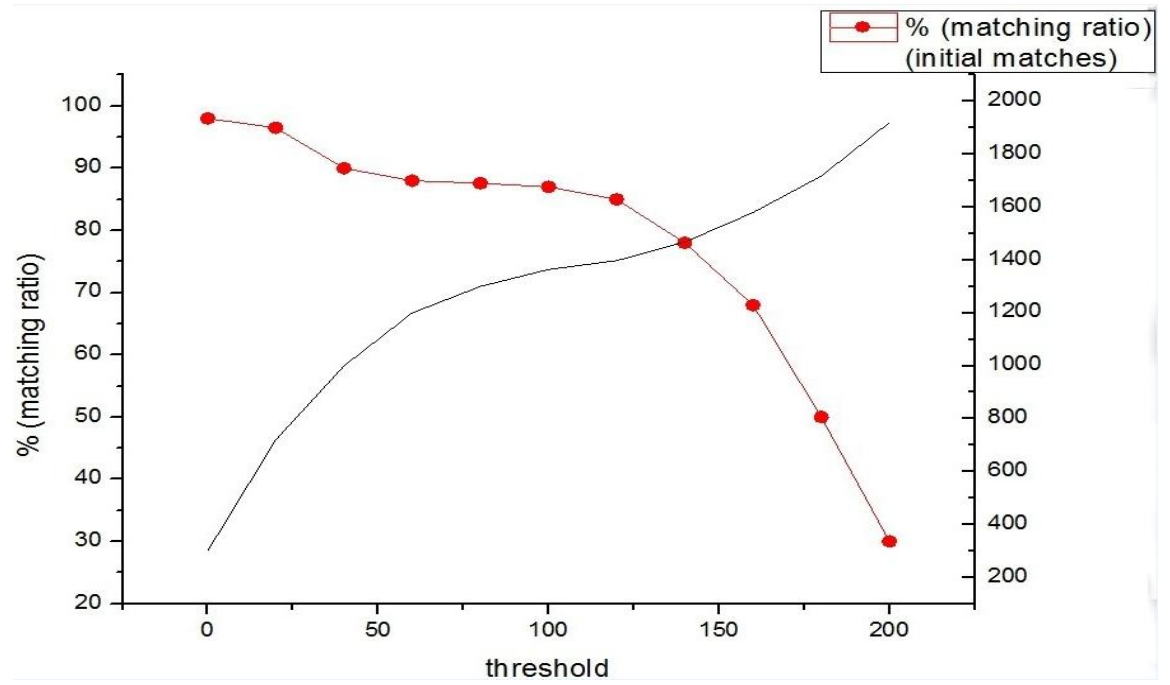

Fig. 4. Threshold (the value of $\tau$ ) affects the initial matches and matching rate.

The pseudo-code of this section described as follow:

$$
\begin{aligned}
& / / S_{O} \text { is the area of original image } \\
& / /\left[R_{L K}, R_{R K}\right] \text { is the Kth block } \\
& / /\left[P_{K L}, P_{K R}\right] \text { is matching pair set of block_k } \\
& / / N u m: \text { The number of blocks } \\
& / / n: \text { The number of matching pairs in block_k } \\
& / / T: \text { The initial transformation matrix } \\
& / / \tau: \text { Euclidean distance threshold }
\end{aligned}
$$




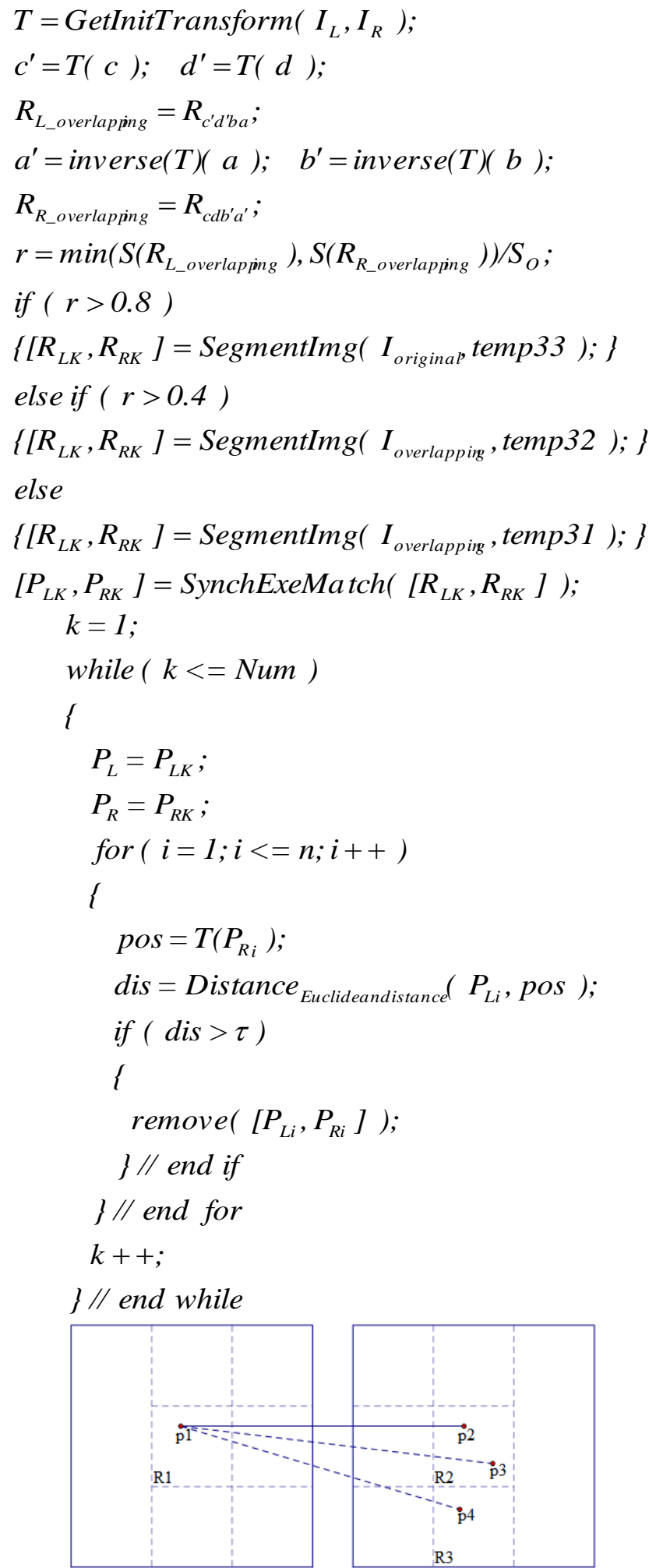

Fig. 5. Eliminate the mismatch through regional division and geometric constraints.

\subsection{Parallel Architecture}

Parallel computing can simultaneously handle multiple tasks or data, and it can significantly improve the efficiency of the processing tasks. Multithreading is a simple and effective way to implement parallel computing. Process contains a number of different execution unit (thread), one of them is the master thread, it is responsible for task allocation and management of the sub thread. When the program is running on multi-core systems, the main thread assign tasks to each child thread after initialization data. Then, sub 
thread is assigned to run on a different CPU. Finally, the master thread collect and process information after all tasks are finished.

To further improve the efficiency of this method, downsampling and blockwise SIFT registration procedure are implemented on the parallel architecture. Firstly, in the coarse registration phase the following steps are implemented on multi-threading: downsampling of the original image pairs (image $I_{L}$ and $I_{R}$ ), feature point extraction, feature descriptor generation. Secondly, the main thread collects feature point set and calculate the initial transformation matrix. And then, image block pairs are sent to the sub thread for blockwise SIFT matching after finish segmenting the overlapping region into several blocks at the master thread. Finally, the master thread collects the matched feature points and computes the fine transform matrix after all tasks are finished.

\subsection{Affine Invariant}

While calculating the principal direction of SIFT descriptor, if the height of the gradient direction is bigger than or equal to $80 \%$ of the principal direction, the direction was added as an auxiliary direction. So these feature points are copied and given the new direction. In this occasion, one feature point may have more than one direction, in other words, one feature point may have several copies. In the process of matching, part or all of these feature points that above mentioned will be become matching points. Actually, they are the same feature point. Hence, these repetitive points should be removed. Bidirectional matching can remove these repetitive points. In addition, SIFT feature vector is a description of the neighborhood of the feature point, it doesn't take into account the global distribution information of feature point and geometric information. Therefore, there are a lot of mismatches resulted from the matching that simply rely on the Euclidean distance between two SIFT vector. In Fig. 6, suppose that we finished matching in source image pairs $\left(I_{L}\right.$ and $\left.I_{R}\right)$, and three pairs of matching points are listed on here $\left\{\left[a_{i}, b_{i}\right],\left[a_{j}, b_{j}\right],\left[a_{k}, b_{k}\right]\right\}$. As shown in Fig. 6, the two triangles are formed from three feature points of image $I_{L}$ and $I_{R}$ respectively. There are translation, rotation and scaling transformation between the two triangles, and they are affine equivalent. Traditional SIFT matching Based on Euclidean distance do not consider this geometric constraints.
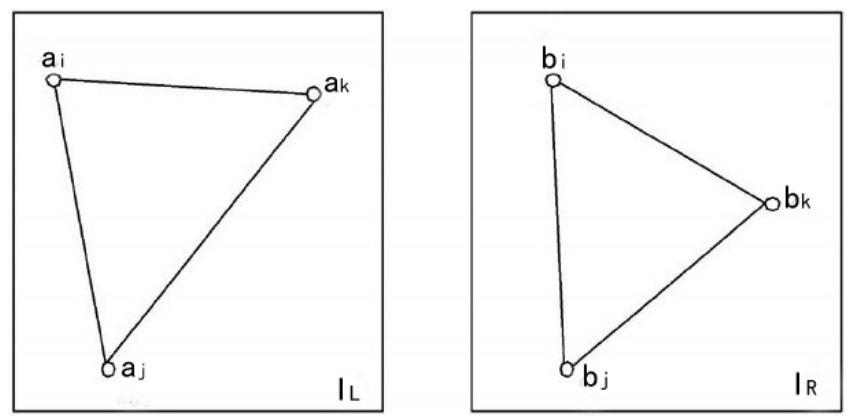

Fig. 6. The two triangles are formed from three feature points of image $I_{L}$ and image $I_{R}$ respectively, and the two triangles are affine equivalent.

In order to eliminate repeat matching and mismatching resulted from above-mentioned two reasons, in this paper, we uses the bidirectional matching. Firstly, Euclidean distance is applied on the positive direction matching. Then, we can further screening the feature points through applying affine invariance of Mahalanobis distance in the opposite direction matching. In this paper, the bidirectional matching algorithm is as follows (the feature points set of $I_{L}$ and $I_{R}$ is $P_{a}$ and $P_{b}$ ): 
1) Search feature points through the BBF algorithm that mentioned in section 2.3, and the similarity measure uses Euclidean distance. Find matching points in $P_{b}$ corresponding to each feature point in $P_{a}$ in proper order, so that we can obtain the initial matching points set

$$
\operatorname{Set}_{\text {first }}=\left\{\left[a_{1}, b_{1}\right],\left[a_{2}, b_{2}\right] \ldots\left[a_{n}, b_{n}\right]\right\} .
$$

The Euclidean distance calculation formula is as follows:

$$
\text { Dis }{ }_{\text {Euclidean }}=\sqrt{\left(a_{i x}-b_{j x}\right)^{2}-\left(a_{i y}-b_{j y}\right)^{2}} .
$$

2) Filtering out the false matches through affine invariants of Mahalanobis distance. Find the matching points in $P_{a}$ corresponding to each feature point in $P_{b}$ in proper order, then, the opposite direction matching points set is obtained,

$$
\operatorname{Set}_{\text {second }}=\left\{\left[b_{1}, a_{1}\right],\left[b_{2}, a_{2}\right] \ldots\left[b_{m}, a_{m}\right]\right\} .
$$

Mahalanobis distance calculation formula is as follows:

$$
D i s_{\text {mahalanobi }}=\sqrt{\left(a_{i}-\mu\right)^{T} C^{-1}\left(a_{i}-\mu\right)},
$$

where

$$
a_{i}=\left(a_{i x}, a_{i y}\right),
$$

Is an sample point in $\mathrm{P}$ and $\mathrm{Q} \mathrm{P}$ and $\mathrm{Q}$ are sample space feature point sets, $a_{i} \in p_{a}$, $\mu$ is the average, $C$ represents the covariance matrix, $\mu$ and $C$ are defined as follows:

$$
\begin{gathered}
\mu=\left(\mu_{x}, \mu_{y}\right)=\frac{1}{n}\left(\sum_{i=1}^{n} a_{i x}, \sum_{i=1}^{n} a_{i y}\right), \\
C=\frac{1}{n}\left[\sum_{i=1}^{n}\left[\begin{array}{l}
a_{i x}-\mu_{x} \\
a_{i y}-\mu_{y}
\end{array}\right]\left[\begin{array}{ll}
a_{i x}-\mu_{x} & a_{i y}-\mu_{y}
\end{array}\right] .\right.
\end{gathered}
$$

3) Calculating the final set of matching points,

$$
\text { Set }_{\text {opt }}=\operatorname{Set}_{\text {first }} \cap \text { Set }_{\text {second }} .
$$

After all, the concrete steps of this paper are:

1) Reduce the source image pairs to low resolution level through direct downsampling, and obtained the image pairs $I_{L^{\prime}}$ and $I_{R^{\prime}}$.

2) Feature Detection. The feature point sets $P_{a}$ and $P_{b}$ of image $I_{L^{\prime}}$ and $I_{R^{\prime}}$ are obtained from SIFT algorithm, and the process is based on multithreading.

3) Initial Matching and Calculate the initial transformation matrix $T$.

4) Apply Section 3.1 to determine the overlapping area of the source image pairs, and segment the overlapping region of two images into several blocks.

5) Extract the SIFT feature points in each block pair based on parallel architecture (multithreaded) described in Section 3.2, apply the third section method to implement fine registration.

6) Collect matched keypoints from each block pair and compute the final Transformation Matrix $T^{\prime}$, then, obtain precise stitching image after resampling. 


\section{Experiments and Discussion}

To assess the precision and the efficiency of the proposed approach in detail, two sets of experiments are designed. The first set of experiments is to verify the registration accuracy of this method, and the second set of experiments aims to show the registration speed of the proposed approach. The experiments are implemented in Matlab and run on Windows 7 system with the computer's processor: Intel (R) Core (TM) 2 Quad CPU 2.83GHz, 4GB of memory. In this paper, the original images used in these experiments shown in Fig. 7:

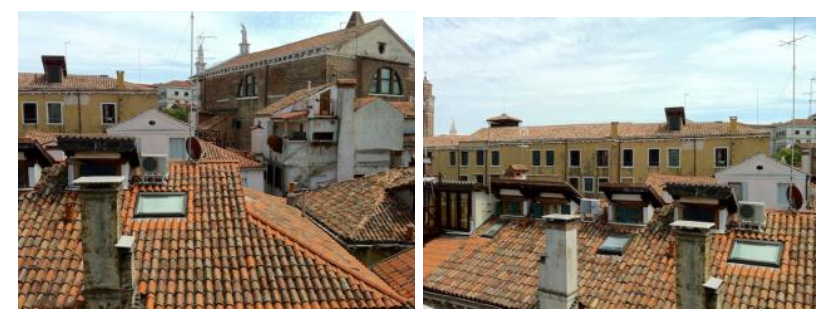

(a)

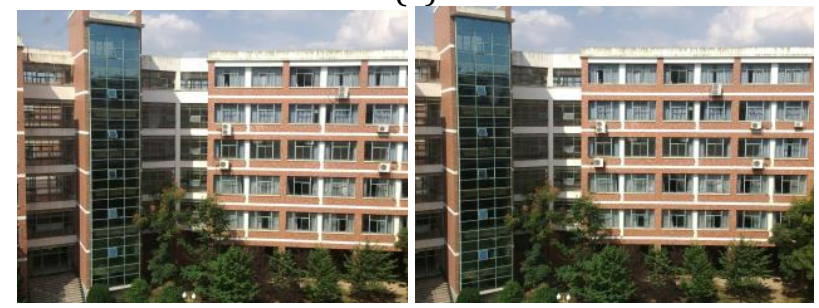

(b)

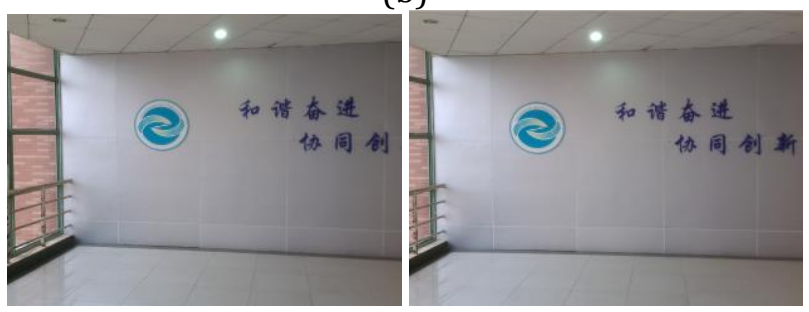

(c)

Fig. 7. The source images.

In order to verify that the block and geometric constraint information can effectively eliminate the mismatching caused by local region similarity, we compared the proposed approach with the traditional SIFT registration method (SIFT + Euclidean distance). In this experiment, we use the matching ratio to evaluate the effectiveness of the block and the geometrical constraint. The computation formula of matching rate is as follows:

$$
R_{\text {match }}=N_{c m} / N_{m}
$$

where, $N_{m}$ is number of matched, $N_{c m}$ is number of correct matches. The experimental results are shown in Fig. 8, and the matching rate of different approaches are listed in Table I.

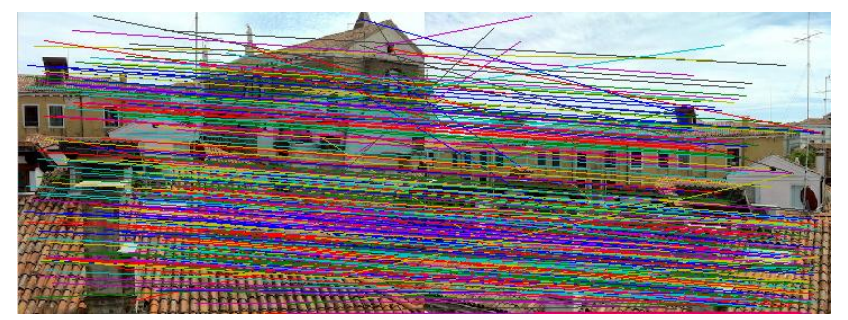

(a1). Matching result based on the traditional SIFT matching approach. 


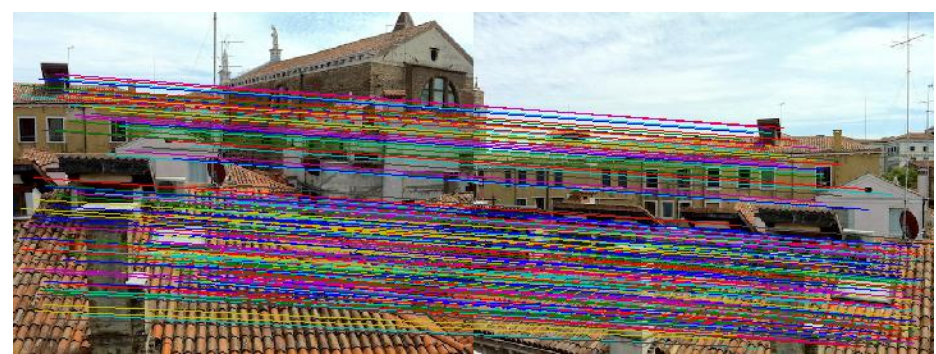

(a2). Matching result based on the proposed approach.

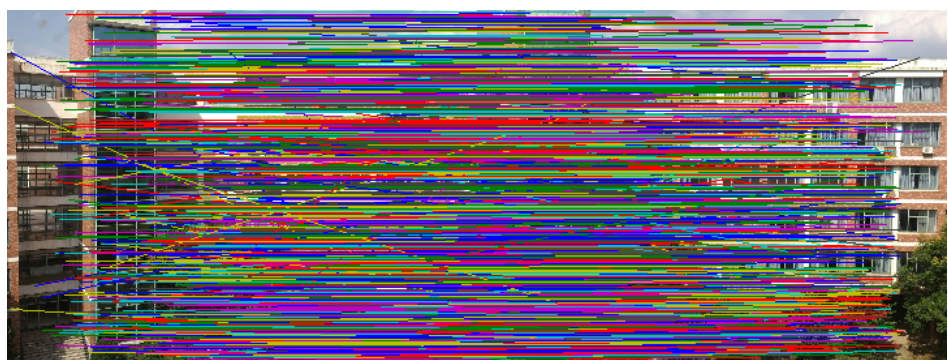

(b1). Matching result based on the traditional SIFT matching approach.

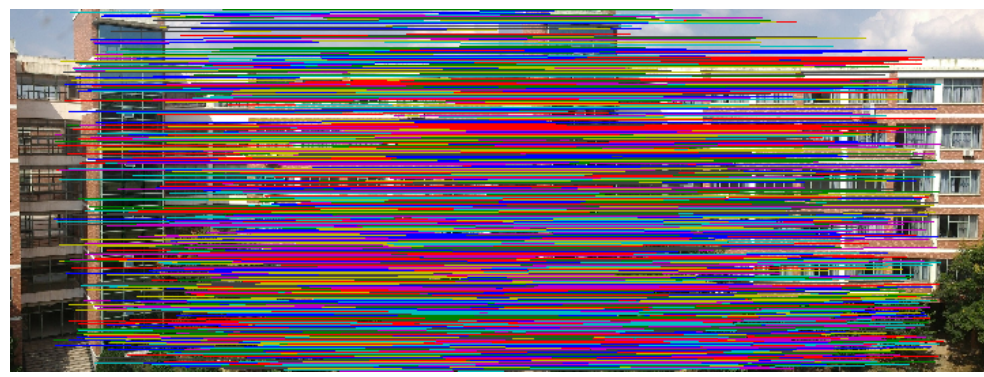

(b2). Matching result based on the proposed approach.

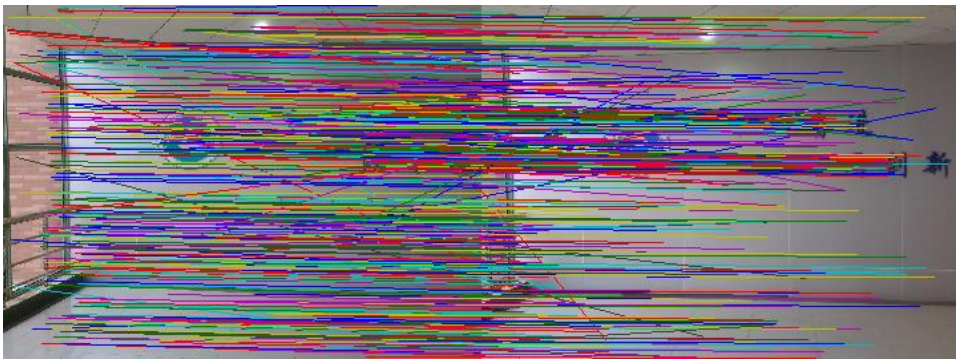

(c1). Matching result based on the traditional SIFT matching approach.

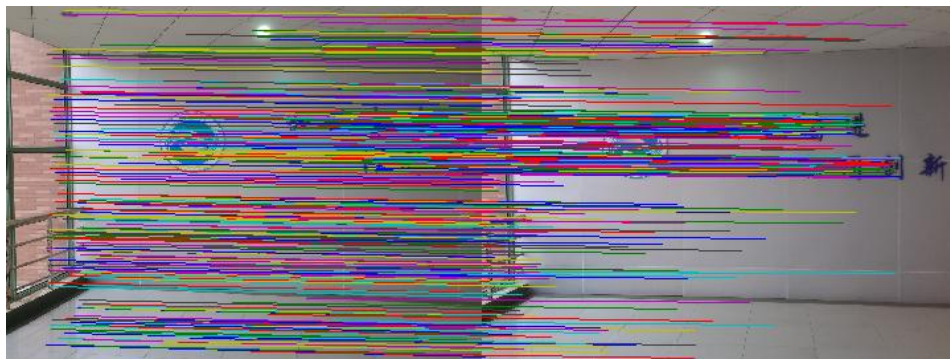

(c2). Matching result based on the proposed approach.

Fig. 8. Matching result.

In Fig. 8(a1)(b1)(c1), we can see that many mismatches obviously. It is because that registration simply rely on the Euclidean distance between two SIFT vectors. And images that exist local region similar or local region not similar but generated SIFT vector similar will result in a close Euclidean distance. Thus, these 
feature points are mistaken for matching point pairs. The proposed approach did not appear this situation after coarse registration and segment the overlapping region. Additionally, we use affine invariants of Mahalanobis distance to further screening the matching points and eliminate mismatches. Thereby, the registration precision is improved significantly. The comparison of the rates of matching is listed in Table 1. For image set a, the traditional SIFT matching approach can achieve 739 pairs, and 335 out of 739 pairs are correct. While the proposed approach can achieve 568 pairs and 326 out of 568 pairs are correct. The matching ratio increases from $45.3 \%$ to $57.4 \%$. For image set $\mathrm{b}$, the matching ratio increases from $79.9 \%$ to $87.2 \%$. For image set c, the matching ratio increases from $60.3 \%$ to $76.5 \%$. The comparisons indicate the advantage of the block and the geometrical constraint in eliminating the false matches.

Table 1. Performance Comparison on Matching Ratio

\begin{tabular}{|c|c|c|c|c|}
\hline Image-set & Method & initial matches & correct matches & matching ratio \\
\hline $\begin{array}{c}\mathrm{a} \\
(\text { Fig.6(a)) } \\
\text { (Fig.7(a1,a2)) }\end{array}$ & $\begin{array}{c}\text { traditional SIFT matching } \\
\text { approach }\end{array}$ & 739 & 335 & $45.3 \%$ \\
\cline { 2 - 4 } $\begin{array}{c}\mathrm{b} \\
\text { (Fig.6(b)) } \\
\text { (Fig.7(b1,b2)) }\end{array}$ & $\begin{array}{c}\text { traditional SIFT matching } \\
\text { approach }\end{array}$ & 1509 & 326 & $57.4 \%$ \\
\cline { 2 - 5 } & the proposed approach & 1364 & 1206 & $79.9 \%$ \\
$\begin{array}{c}\text { (Fig.6(c)) } \\
\text { (Fig.7(c1,c2) }\end{array}$ & $\begin{array}{c}\text { traditional SIFT matching } \\
\text { approach }\end{array}$ & 892 & 538 & $87.2 \%$ \\
\cline { 2 - 5 } & the proposed approach & 689 & 527 & $76.5 \%$ \\
\hline
\end{tabular}

Table 2. Performance Comparison at Registration Time

\begin{tabular}{|c|c|c|c|c|}
\hline Image-set & $\begin{array}{c}\text { the traditional SIFT } \\
\text { registration approach } \\
\text { based on the single thread }\end{array}$ & $\begin{array}{c}\text { the proposed } \\
\text { approach based on the } \\
\text { single thread }\end{array}$ & $\begin{array}{c}\text { the proposed approach } \\
\text { (based on } \\
\text { multithreading) }\end{array}$ \\
\hline a & time(s) & 2.1722 & 3.7212 & 1.3589 \\
\hline b & time(s) & 5.2155 & 6.9896 & 3.5365 \\
\hline c & time(s) & 3.4697 & 4.9567 & 2.2536 \\
\hline
\end{tabular}

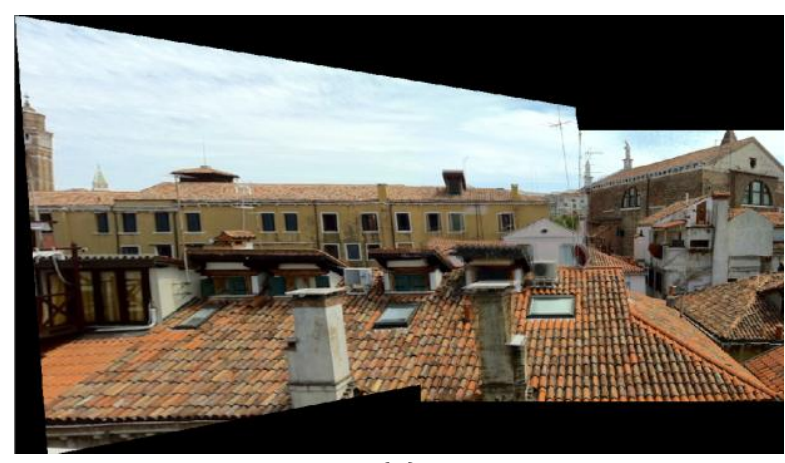

(a)

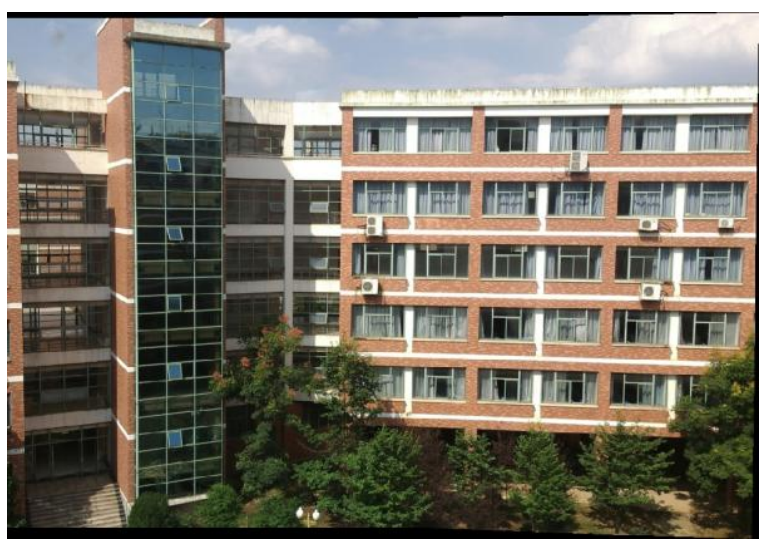

(b) 


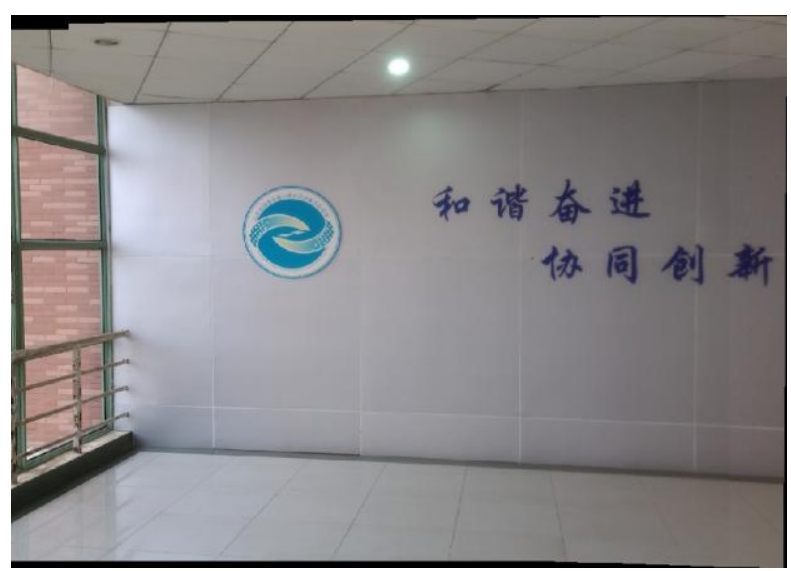

(c)

Fig. 9. Mosaic result by the proposed approach.

To verify the registration efficiency of the proposed approach, we compared the execution time of these three methods: the proposed approach, the traditional SIFT matching approach based on the single thread, and the proposed approach that based on the single thread. The results are shown in Table 2.

From Table 2, we can see that the registration efficiency of the proposed approach is improved significantly. For image set a, the execution time reduces from 2.1722 to 1.3589 . For image set $b$, the execution time reduces from 5.2155 to 3.5365. For image set c, the execution time reduces from 3.4697 to 2.2536. And this demonstrates the advantages of multithreading in improving the efficiency. The registration time of the proposed approach based on single thread is longer than that of the traditional SIFT registration method based on single thread. This is because the method spend more time in the following aspects: coarse registration, regional division, using the geometric constraints and affine invariants screening the matching points. The proposed method makes full use of the advantages of parallel, and it improves the efficiency of the registration significantly. The following processes are completed based on multi-threading: downsampling of the original image pairs, Establishment of a Gaussian pyramid, Feature point extraction, and the fine registration of each block pair.

We calculate the final transformation matrix through the matching point set after finishing fine registration. Then, through image resampling, the result of image stitching are shown in Fig. 9.

\section{Conclusion}

With the help of geometric constraints and parallel architecture, a new effective image registration approach is proposed and implemented. This method has solved these two problems that mismatch problems which are resulted by images which have local region similarities and spend too long time on registration. First of all, in order to provide geometric constraints for block and fine registration, we use SIFT algorithm parallelly extract feature points to calculate initial transform matrix. Then, segment the overlapping region of original image pairs into several blocks. Finally, to achieve fine registration, blockwise SIFT matching and eliminate the mismatch through geometric constraints are implemented on parallel architecture. During the fine registration, we use geometric constraints and affine invariance of Mahalanobis distance to screen feature points further and eliminate duplicate matches and mismatches to improve the matching accuracy. The experimental results show that this method has eliminated the mismatch generated by same local features. Moreover, registration accuracy and speed have been improved, the registration rate increased from $45.3 \%$ to $57.4 \%$ and time reduced from 2.1722 s to $1.3589 \mathrm{~s}$. Our method also requires improvements in the following respect: the template used in overlapping regional division have not thoroughly explored how to divide the overlapping region to further improve the 
registration rate, and therefore we need to study how to select the number of blocks self-adaptively according to the sizes of the image resolution and overlapping region.

\section{Acknowledgment}

This paper is sponsored by Nature Science Foundation of China (61272216) and innovation Foundation of aerospace in China (CASC201102).

\section{References}

[1] Izadi, M., \& Saeedi P. (2012). Robust weighted graph transformation matching for rigid and nonrigid image registration. IEEE Transactions on Image Processing, 21(10),4369-4382.

[2] Lin, Z., Deng, D. X., Chen, X., \& Zhang, Y. L. (2012). A self-adaptive and real-time panoramic video mosaicing system. Journal of Computers, 7(1), 218-225.

[3] Zhu, Z. X., \& Wang, G. Y. (2014). Shape-matching model optimization using discrete-point sampling and feature salience. Journal of Computers, 9(6), 1355-1363.

[4] Chiu, L. C., Chang, T. S., Chen, J. Y., \& Chang, N. Y. (2013). Fast SIFT design for real-time visual feature extraction. IEEE Trans Image Process, 22(8), 3158-3167.

[5] Fan, B., Huo, C. L., Pan, C. H., \& Kong, Q. Q. (2013). Registration of optical and SAR satellite images by exploring the spatial relationship of the improved sift. IEEE Geoscience and Remote Sensing Letters, 10(4), 657-661.

[6] Hye, W. K., \& Suk, I. Y. (2014). Defect detection using feature point matching for non-repetitive patterned images. Pattern Analysis and Applications, 17(2), 415-429.

[7] Cheng, P., \& Menq, C. H. (2013). Real-time continuous image registration enabling ultraprecise 2-D motion tracking. IEEE Trans Image Process, 22(5), 2081-2090.

[8] Dong, T. Z., Deng, T. Q., Dai, J. S., Xie, W., \& Yang, J. H. (2013). A fuzzy reasoning method for multi-views image registration. Journal of Computers, 8(10), 2689-2694.

[9] Sun, W., Wiro, J., Niessen, M. V. S., \& Stefan, K. (2013). Simultaneous multiresolution strategies for nonrigid image registration. IEEE Transactions on Image Processing, 22(12), 4905-4917.

[10] Harris, C., \& Stephens, M. A. (1998). Combined corner and edge detector. Proceedings of the 4th Alvey Vision Conference.

[11] Lowe, D. (2004). Distinctive image features from scale-invariant key points. International Journal of Computer Vision, 60(2), 91-110.

[12] Herbert, B., Tinne, T., \& Luc, V. G. (2006). Speeded up robust features. Proceedings of the Computer Vision Lecture Notes in Computer Science (pp. 404-417).

[13] Herbert, B., Andreas, E., Tinne, T., \& Luc, V. G. (2008). Speeded-up robust features. Computer Vision and Image Understanding, 110(3), 346-359.

[14] Liu, L., Peng, F. Y., Zhao, K., \& Wan, Y. P. (2008). Simplified SIFT algorithm for fast image matching. Infrared and Laser Engineering, 37(1),181-184.

[15] Ke, Y., \& Sukthankar, R., A more distinctive representation for local image descriptors. Proceedings of the 2004 IEEE Computer Society Conference on Computer Vision and Pattern Recognition (pp. 506-513).

[16] Yan, W. W., \& Hui, L. Y. (2013). Image registration method based on PCA-SIFT feature detection. Advanced Materials Research, 2395-2398.

[17] JI, H., Wu, Y. H., Sun, H. H., \& Wang, Y. J. (2009). SIFT feature matching algorithm with global information. Optics and Precision Engineering,17(2),439-444.

[18] Bastanlar, Y., \& Temizel, A., (2010). Improved SIFT matching for image pairs with scale difference. Electronics Letters, 46(5), 346-348. 
[19] Goncalves, H., Corte, Real, L., \& Goncalves, J. (2011). Automatic image registration through image segmentation and sift. Geoscience and Remote Sensing, 49(7), 2589-2600.

[20] Qiang, S., Guorui, M., Feifei, Z., Wangli, C., \& Qianqing, Q. (2014). Robust image registration using structure features. IEEE Geoscience and Remote Sensing Letters, 11(12), 2045-2049.

[21] Kai, Z., Xu, Z. L., \& Jiu, X. Z. (2014). A robust point-matching algorithm for remote sensing image registration. IEEE Geoscience and Remote Sensing Letters, 11(2), 469-473.

[22] Jin, Y., Anders, E., Tat, J. C., \& David, S. (2014). An adversarial optimization approach to efficient outlier removal. Journal of Mathematical Imaging and Vision, 48(3), 451-466.

[23] Wang, Y, X. \& Yan, M. (2012). A method of line matching based on feature points. Journal of Software, $7(7), 1539-1545$.

Zetao Jiang was born in 1961 in Jiangxi Province. He is adjunct professor of Guilin Uniersity of Electronic Technology, PhD supervisor, academic leader in Guangxi province. He received his B.S. degree in Beijing Normal University, Beijing, China, in 1986. He received his M.S. degree in Tongji University, Shanghai, China, in 1995 and received the doctor of philosophy in Northwestern Polytechnical University, Xi'an, China, in 2006. His research interests include image processing and computer vision.

He presided key technology research project "Control system based on multi-level security agent", which was awarded the second class prize in the 2009 annual science and technology progress of Jiangxi province. He presided the research of network and information security defense technology based on digital watermarking, which was awarded the first class prize in science and technology achievements of Jiangxi province. He presided and finished Nature Science Foundation of China(60673055), two subject Foundations of province, an open foundation of measurement and control center of province and more than 10 other subjects. Currently, he is presiding Nature Science Foundation of China(60673055) and Nature Science Foundation of Province, which is in the field of 3D reconstruction.

Chuan Guo was born in Chongqing Municipality, in 1990. He received his B.S degree in Nanchang Hang Kong University, Nanchang, China, in 2012. He was recommended for admission to pursue a master's degree, in 2012. Currently, he is pursuing the M.S degree in Nanchang Hang Kong University. His research interests include image processing and computer vision.

Yang Yang was born in Jiangxi Province, in 1989 and He received his B.S degree in Nanchang Hang Kong University, Nanchang, China, in 2012. Currently, he is also pursuing the M.S degree in Nanchang Hang Kong University, and His research interests include image processing and computer vision. 